\title{
CORRELAÇÃO ENTRE A QUALIDADE DE VIDA DE CUIDADORES FAMILIARES E OS NÍVEIS DE INDEPENDÊNCIA FUNCIONAL DOS CUIDADOS
}

Renato Nickel ${ }^{1}$, Andressa Pereira Lima², Elaine Janeczko Navarro² ${ }^{2}$ Lauren Machado Pinto², Hélio Afonso Ghizoni Teive $^{3}$, Nilson Becker ${ }^{4}$

RESUMO: A pesquisa correlacionou o nível de Independência Funcional de pessoas com sequelas de doenças neurológicas com a Qualidade de Vida (QV) dos cuidadores principais. Para tanto, utilizou a Medida de Independência Funcional (MIF) com 40 sujeitos e o Short-Form Health Survey (SF-36) com 40 cuidadores. As variáveis levantadas não apresentaram significância estatística $\mathrm{p}<0,05$. Comparando os escores da MIF com a QV, apenas uma pequena correlação entre independência funcional e aspectos sociais para $\mathrm{p}<0,05$. Correlacionando a QV com os níveis de independência, para os sujeitos com moderada dependência, apresentou uma alta correlação (Spearman) para Capacidade Funcional p<0,01; e para os sujeitos completamente dependentes, uma alta correlação (Spearman) para Dor e Saúde Mental p<0,01, e para Estado Geral da Saúde e Vitalidade $\mathrm{p}<0,05$. Os dados mostram a necessidade de uma abordagem específica sobre a QV de cuidadores de sujeitos que apresentam uma dependência completa.

PALAVRAS-CHAVE: Assistência domiciliar; Terapia ocupacional; Pessoas com deficiência; Qualidade de vida.

\section{CORRELATION BETWEEN THE CAREGIVERS' QUALITY OF LIFE AND FAMILY LEVELS OF FUNCTIONAL INDEPENDENCE OF CARE}

\begin{abstract}
The research correlated the Functional Independence level of people with neurological sequels, with the caregivers' Quality of Life (QOL). The Functional Independence Measure (FIM) was used with 40 subjects and the ShortForm Health Survey (SF-36) with 40 caregivers. The variables studied were not statistically significant $\mathrm{p}<0.05$. Comparing the scores of FIM with QOL, only a small correlation between functional independence and social aspects to $\mathrm{p}<0.05$. Correlating QOL with levels of independence, individuals with moderate dependence showed a high correlation (Spearman) for functional capacity $\mathrm{p}<0.01$, and a high correlation (Spearman) for the subjects completely dependent on for Pain and Mental Health $\mathrm{p}<0.01$ and General Health and Vitality $\mathrm{p}<0.05$. Data show the need for a specific approach on the QOL of caregivers of individuals who have a complete dependence.
\end{abstract}

KEYWORDS: Home care; Occupational therapy; People with disabilities; Quality of life.

\section{CORRELACIÓN ENTRE LA CALIDAD DE VIDA DE CUIDADORES FAMILIARES Y LOS NIVELES DE INDEPENDENCIA FUNCIONAL DE LOS CUIDADOS}

RESUMEN: La investigación correlacionó el nivel de Independencia Funcional de personas con secuelas de enfermedades neurológicas con la Calidad de Vida (QV) de los cuidadores principales. Para tanto utilizó la Medida de Independencia Funcional (MIF) con 40 sujetos y/o Short-Form Health Survey (SF-36), con 40 cuidadores. Las variables levantadas no presentaron significancia estadística $\mathrm{p}<0,05$. Comparando la puntuación de la MIF con la QV, apenas una pequeña correlación entre Independencia funcional y aspectos sociales para $\mathrm{p}<0,05$. Correlacionando la QV con los niveles de Independencia, para los sujetos con moderada dependencia presentó una alta correlación (Spearman) para Capacidad Funcional $\mathrm{p}<0,01 \mathrm{y}$, para los sujetos completamente dependientes una alta correlación (Spearman) para Dolor y Salud Mental p<0,01 y para Estado General de la Salud y Vitalidad $\mathrm{p}<0,05$. Los datos muestran la necesidad de un abordaje específico sobre la QV de cuidadores de sujetos que presentan una dependencia completa.

PALABRAS CLAVE: Atendimiento domiciliario; Terapia ocupacional; Personas con deficiencia; Calidad de vida.

\footnotetext{
${ }^{1}$ Terapeuta Ocupacional. Doutor em Educação. Professor Adjunto do Curso de Graduação em Terapia Ocupacional da Universidade Federal do Paraná-UFPR

${ }^{2}$ Acadêmica do Curso de Graduação em Terapia Ocupacional da UFPR.

${ }^{3}$ Médico Neurologista. Professor Adjunto do Curso de Medicina da UFPR. Chefe do Ambulatório de Distúrbio de Movimento do Hospital de Clínicas-HC/UFPR.

${ }^{4}$ Médico Colaborador do Ambulatório de Distúrbio de Movimento do HC/UFPR.
}

Autor correspondente:

Renato Nickel

Universidade Federal do Paraná

Rua Padre Camargo, 280 - 80060-240 - Curitiba-PR, Brasil

Recebido: 14/09/09

E-mail: renatonickel@hotmail.com

Aprovado: 17/05/10 


\section{INTRODUÇÃO}

Qualidade de Vida (QV) é um conceito culturalmente construído, influenciado pelo tempo, lugar, fatores socioculturais, biológicos e psicológicos ${ }^{(1)}$, através de uma visão holística que engloba os aspectos sociais, emocionais e de bem-estar físico e material, por meio das percepções subjetivas de cada indivíduo em relação ao meio em que vive e das suas expectativas acerca desse meio. Ou seja, QV é um conceito construído por cada um, tornando difícil defini-lo de um modo generalizado, daí a importância da utilização de instrumentos padronizados para a pesquisa desse tema com uma população numerosa ${ }^{(2)}$.

O cuidar perpassa o conceito de QV, tanto no que diz respeito ao cuidar de si quanto ao cuidar de outrem. No segundo caso, quando ocorre a dependência funcional de um sujeito, esta mobiliza não só demandas individuais, mas também familiares e sociais, as quais afetam a rotina e a estrutura em que o sujeito está inserido, ocorrendo, desta forma, mudanças de papéis, financeiras, entre outras ${ }^{(3)}$.

A vida cotidiana é formada por relações sociais, nas quais os seres humanos se inserem desde o nascimento, criando assim rotinas através das representações culturais do grupo de inserção $0^{(4)}$. Assumir o cuidado é uma situação que perpassa a obrigação imposta, ora pelo sistema social, ora pelo emocional do cuidador, que muitas vezes se sobrecarrega e se expõe a riscos de comprometimento de sua própria saúde, em beneficio do doente ${ }^{(5)}$.

Um dos fatores que predispõem o papel de cuidador é a ocorrência cada vez mais freqüente de doenças neurológicas, como o Acidente Vascular Encefálico (AVE) que, em muitos casos, deixa sequelas permanentes e incapacitantes, solicitando assim a presença constante do cuidador como fator central do atendimento desses sujeitos ${ }^{(5)}$.

$\mathrm{Na}$ realidade brasileira, devido a fatores socioeconômicos das famílias, o papel de cuidador agente fundamental em todo o processo de reabilitação - é gerado dentro do próprio contexto familiar, situação que acaba sobrecarregando o sofrimento da família e, consequentemente, levando a desvios de funções intrafamiliares.

O cuidador familiar torna-se um sujeito sensível devido a mudanças de seus próprios papéis na família, condição que necessita de atenção, pois o cuidador sofre não somente com a limitação daquele que requer cuidados, mas também pelas múltiplas funções assumi- das, ou seja, por aquelas que não consegue desempenhar da forma como o fazia e mesmo aquelas que não mais poderá desempenhar ${ }^{(6)}$.

Desta forma, a partir dessa relação tênue entre a quantidade de auxílio que um sujeito dependente necessita durante seu dia-a-dia e a organização do cuidador e da família em relação à atividade de cuidar, buscou-se neste trabalho correlacionar o nível de dependência de quem é cuidado com a QV de quem cuida, com o objetivo de descrever de forma mais criteriosa esta relação.

A importância da atenção em saúde para os cuidadores, em vários contextos de atendimento, bem como a necessidade de orientações de profissionais da saúde para que possam fornecer o melhor cuidado possível durante o processo de reabilitação do sujeito, foram os incentivadores desse projeto.

Na literatura há ampla referência sobre QV e independência funcional de sujeitos que apresentam condições crônicas de saúde. No entanto, em levantamentos realizados na literatura nacional, na base de dados BIREME, não foram encontradas referências sobre a relação entre estes dois níveis, se um interfere sobre o outro, ou mesmo se existem outras variáveis influentes nessa relação.

Desta forma, o estudo se justifica devido à escassez de referencial bibliográfico acerca da correlação QV e dependência funcional, tendo em vista fortalecer as ações voltadas a essa população, a qual cresce cada dia mais em nosso país e é carente, muitas vezes, de orientações e de um acompanhamento profissional domiciliar adequado.

\section{METODOLOGIA}

Este é um estudo exploratório descritivo do tipo transversal e foi realizado no Ambulatório de Espasticidade do Hospital de Clínicas da Universidade Federal do Paraná (HC-UFPR), de setembro a dezembro de 2007. O Ambulatório de Espasticidade é um serviço estruturado onde os sujeitos participantes são sequelados devido a doenças neurológicas relacionadas à Síndrome do Neurônio Motor Superior. Esta síndrome gera, na maioria dos casos, deficiências nas funções corporais e, consequentemente, incapacidade para o desempenho de tarefas de vida diária.

Para o estudo foram recrutados 80 participantes. Destes, 40 pacientes eram do referido ambulatório, sendo critério de inclusão apresentar condições crônicas de saúde oriundas de doenças neurológicas, 
e critérios de exclusão apresentar as mesmas condições, mas com incapacidade de cognição. Os outros 40 sujeitos foram seus respectivos cuidadores, selecionados a partir do critério de ser o cuidador principal, ou seja, prestar cuidados aos sujeitos em mais de $50 \%$ do tempo. Outro critério de inclusão para ambos os participantes foi aceitar participar da pesquisa e assinar do Termo de Consentimento Livre e Esclarecido.

Para avaliação da funcionalidade dos sujeitos foi utilizada a Medida de Independência Funcional (MIF), que aborda 18 tarefas referentes ao autocuidado, controle esfincteriano, transferências, locomoção, comunicação e a cognição social. A pontuação se dá de 1 a 7 , que reflete uma escala em que 1 é o maior nível de dependência e 7 o menor nível de dependência, ou seja, 7 caracterizaria um sujeito independente ${ }^{(3)}$. Contudo, para melhor visualização dos resultados, dividimos o nível de dependência da MIF em três níveis: Sujeitos com Independência, Sujeitos com Dependência Moderada e Sujeitos com Dependência Completa.

Para avaliação da QV dos cuidadores foi utilizada a SF-36 (Medical Outcomes Study 36 - Item Short-Form Health Survey) que é um instrumento traduzido e validado no Brasil, composto por 36 itens que fornecem pontuações em oito dimensões da qualidade de vida, sendo elas: Capacidade Funcional (CF); Aspectos Físicos (AF); Dor (D); Estado Geral da Saúde (EGS); Vitalidade (V); Aspectos Sociais (AS); Aspectos Emocionais (AE); e Saúde Mental (SM). A pontuação varia entre 0 e 100 pontos, que demonstram a pior e a melhor QV possível ${ }^{(7)}$, respectivamente.

As avaliações foram realizadas após o atendimento ambulatorial, sendo a MIF feita junto aos sujeitos sequelados crônicos na presença dos cuidadores, e o SF-36 aplicada junto aos cuidadores, sem a presença daqueles que eram cuidados, tendo duração média de 30 minutos cada.

Além da MIF e do SF-36, foram coletados dados sobre gênero, estado matrimonial, ocupação atual, tipo de cuidado, condição de saúde, idade e educação formal.

As médias e desvio padrão do SF-36 encontrados para os cuidadores foram comparados, primeiramente, com as médias encontradas na literatura para pessoas com condições normais de saúde, caracterizando a população em geral, e posteriormente foram correlacionadas com os resultados da MIF.

A pesquisa foi aprovada pelo Comitê de Ética em Pesquisa do Hospital de Clínicas da Universidade
Federal do Paraná, sob o registro 1524.189/2007-09.

\section{RESULTADOS E DISCUSSÃO}

Com relação às características gerais da amostra, os cuidadores eram, em sua maioria, mulheres (80\%), sendo em sua totalidade cuidadores familiares, com média de idade de 48 anos $( \pm 10,93)$, com média de 9,2 anos $( \pm 5,04)$ de educação formal. Com relação ao estado matrimonial dos cuidadores, $70 \%$ estavam casados, e 45\% exerciam atividade laboral assalariada; todos os cuidadores apresentaram grau de parentesco próximo de quem recebia os cuidados, sendo que $62,5 \%$ eram mães.

Já com relação aos sujeitos que recebiam os cuidados, os dados descritivos se apresentaram da seguinte maneira: $57,5 \%$ eram do sexo masculino e $42,5 \%$ do sexo feminino; a média de idade foi de 36,3 anos $( \pm 17,58)$; a média de anos de educação formal foi de 6,77 anos $( \pm 4,60)$; $62,5 \%$ eram solteiros (nunca foram casados); 35\% eram aposentados; e os diagnósticos mais presentes foram os de Paralisia Cerebral (PC) 32,5\%, e Acidente Vascular Encefálico (AVE) com 22,5\%.

No que diz respeito à literatura voltada aos cuidadores, os resultados encontrados no presente estudo seguem a mesma linearidade histórica, que traz a mulher como principal cuidadora ${ }^{(3)}$, atribuição esperada pela sociedade ${ }^{(5)}$.

Em relação à QV dos cuidadores, através dos 8 domínios avaliados pela SF-36, encontramos: média de 82,63 $(25,03)$ para Capacidade Funcional (CF); 70,62 $( \pm 32,46)$ para Aspectos Físicos (AF); 60,08 $( \pm 20,67)$ para Dor; $77,27( \pm 18,01)$ para Estado Geral da Saúde (EGS); 66,13 ( $\pm 20,67)$ para Vitalidade; 74,38 $( \pm 23,67)$ para Aspectos Sociais (AS); 72,50 ( $\pm 35,31)$ para Aspectos Emocionais (AE); e 71,10 $( \pm 20,00)$ para Saúde Mental (SM). Todos os domínios apresentamse diminuídos, visto que a melhor QV possível é 100; entretanto, foi observada uma diminuição acentuada nos domínios Dor e Vitalidade. Estes dois domínios estão corroborados pela literatura, que traz a sobrecarga como principal situação encontrada na função de cuidador. Este vivencia o sofrimento ao conviver com a doença do familiar, seja pela tensão e cansaço, seja pelo lazer comprometido pela tarefa de cuidar $^{(5)}$.

Na tabela 1 comparamos os domínios da SF-36 encontrados pelo presente estudo com os domínios encontrados por Morales e colaboradores ${ }^{(8)}$, em seu 
Tabela 1 - Comparação da QV dos cuidadores com a QV da População em Geral. Curitiba, 2007.

\begin{tabular}{lcccc}
\hline & \multicolumn{2}{c}{$\begin{array}{c}\text { Presente Estudo } \\
\text { QV dos Cuidadores }\end{array}$} & \multicolumn{2}{c}{$\begin{array}{c}\text { Morales et al (2007) } \\
\text { QV da População em Geral }\end{array}$} \\
\hline Domínios da SF-36 & Média 1 & $\begin{array}{c}\text { Std. } \\
\text { Deviation 1 }\end{array}$ & Média 2 & $\begin{array}{c}\text { Std. } \\
\text { Deviation 1 }\end{array}$ \\
\hline Capacidade Funcional & 82,63 & 25,03 & 94,90 & 8,00 \\
Aspectos Físicos & 70,62 & 32,46 & 94,60 & 14,10 \\
Dor & 60,08 & 20,67 & 87,80 & 12,50 \\
Estado Geral de Saúde & 77,27 & 18,01 & 87,60 & 10,90 \\
Vitalidade & 66,13 & 20,67 & 78,00 & 14,60 \\
Aspectos Sociais & 74,38 & 23,67 & 89,70 & 15,50 \\
Aspectos Emocionais & 72,50 & 35,31 & 88,40 & 26,10 \\
Saúde Mental & 71,10 & 20,00 & 78,70 & 15,70 \\
\hline
\end{tabular}

grupo controle, ao descrever a QV de sujeitos com Esclerose Múltipla, quando utilizaram, para comparação e controle, sujeitos doadores de sangue.

A população do presente estudo, em comparação ao estudo apresentado ${ }^{(8)}$ em referência à população em geral, apresenta valores menores em todos os domínios da SF-36. Assim, é possível observar que os cuidadores avaliados têm a QV diminuída, principalmente em relação aos domínios de Dor, Aspectos Físicos, Aspectos Sociais e Aspectos Emocionais.

Com relação à dependência funcional dos sujeitos que recebem os cuidados, de acordo com a MIF, 17 apresentaram independência (42,5\%); 12 nível de dependência moderada (30\%); e 11 apresentaram o nível de dependência completa (27,5\%). As variáveis levantadas não foram estatisticamente significantes para $\mathrm{p}<0,05$. Na correlação (Spearman) entre os dados pessoais e a QV dos cuidadores, não foram encontradas correlações estatisticamente significativas. Entretanto, encontramos uma pequena correlação entre a MIF de todos os sujeitos que receberam cuidados e Aspectos Sociais dos cuidadores para $\mathrm{p}<0,05$.

Na tabela 2 podemos verificar as correlações entre os níveis de dependência dos sujeitos e os domínios da QV dos cuidadores. Quando correlacio-

Tabela 2 - Correlação entre níveis de dependência dos sujeitos e domínios da QV dos cuidadores. Curitiba, 2007.

\begin{tabular}{|c|c|c|c|}
\hline \multirow[t]{2}{*}{ Domínios do SF-36 } & \multicolumn{3}{|c|}{ Spearman Correlation } \\
\hline & Independência & Dependência Moderada & Dependência Completa \\
\hline Capacidade Funcional & 0,054 & $0,722 * *$ & $-0,392$ \\
\hline Aspecto Físico & 0,410 & 0,298 & $-0,569$ \\
\hline Dor & 0,055 & 0,061 & $-0,867$ \\
\hline Estado Geral de Saúde & 0,159 & $-0,288$ & $-0,680 * *$ \\
\hline Vitalidade & 0,523 & $-0,069$ & $-0,652^{*}$ \\
\hline Aspectos Sociais & 0,247 & 0,00 & $-0,445$ \\
\hline Aspectos Emocionais & 0,467 & $-0,124$ & $-0,315$ \\
\hline Saúde Mental & 0,023 & $-0,195$ & $-0,797 * *$ \\
\hline
\end{tabular}

*Correlação significante a 0,05

**Correlação significante a 0,01

namos a QV com a MIF, de acordo com o nível de dependência do sujeito, não encontramos correlação significativa para os sujeitos independentes.

Encontramos, para os sujeitos com moderada 
dependência, uma alta correlação (Spearman) entre a MIF e a Capacidade Funcional para $\mathrm{p}<0,01$. Para os sujeitos completamente dependentes, encontramos uma alta correlação (Spearman) entre MIF, Dor e Saúde Mental para p $<0,01$ e entre MIF, Estado Geral da Saúde e Vitalidade para $\mathrm{p}<0,05$.

Na comparação entre os níveis da MIF e os domínios da SF-36 (Gráfico 1), observa-se que as médias dos domínios apresentam diminuição significativa entre os cuidadores de sujeitos que se apresentam mais independentes em relação aos sujeitos que necessitam de maiores cuidados e estão enquadrados no nível

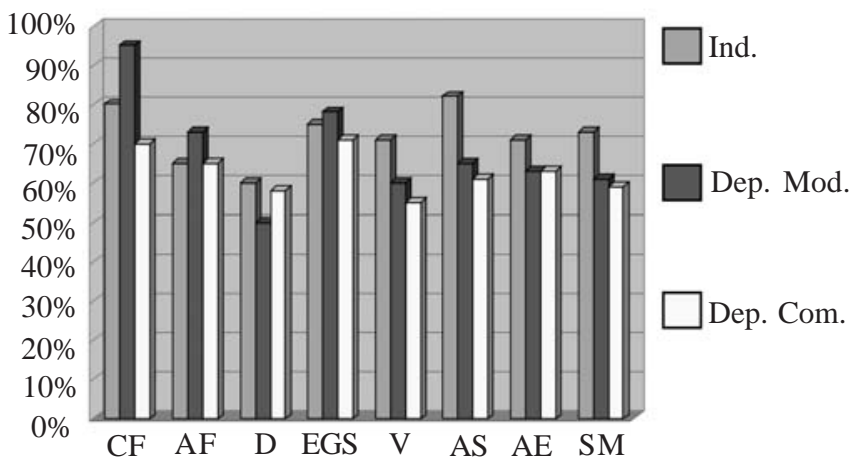

Gráfico 1 - Comparação entre os domínios da SF-36 e os níveis de dependência da MIF. Curitiba, 2007.

Legenda: Ind. - Independência; Dep. Mod - Dependência Moderada; Dep. Com. - Dependência Completa; CF - Capacidade Funcional; AF - Aspectos Físicos; D - Dor; EGS - Estado Geral de Saúde; V - Vitalidade; AS - Aspectos Sociais; AE - Aspectos Emocionais; SM - Saúde Mental

de dependência completa. Essa relação apenas não está presente no domínio Dor, em que o valor apresenta menor escore no nível de dependência moderada.

Um aspecto importante a ser salientado é que quando o tema QV é estudado, o quesito "renda familiar" é um aspecto importante a ser analisado, pois renda familiar mais alta pode determinar melhores condições de vida. No presente estudo, a renda familiar não foi coletada individualmente. Contudo, entende-se que devido ao contexto da coleta de dados ser o de um hospital público e com a totalidade dos atendimentos vinculados ao Sistema Único de Saúde, a população atendida se enquadraria na faixa média salarial da população brasileira, aquela que utiliza o Sistema Público de Saúde, ou seja, de baixa renda.

\section{CONSIDERAÇÕES FINAIS}

O cuidador é a pessoa que se envolve no processo de cuidar do outro, com qualidades pessoais e, muitas vezes, com forte traço de amor. Ele realiza ações como cuidados de higiene, administração de medicamentos, cuidados com a pele, troca de curativos, procedimentos invasivos e cuidados com sondas de alimentação $0^{(9)}$. Estas situações perpassam até mesmo a relação de amorosidade, acarretando a aquisição de novos papéis ocupacionais, sobrecarga e a diminuição da QV do cuidador. Isso se verifica principalmente quando - como na população estudada - os cuidadores se configuram, em sua totalidade, como cuidadores familiares, mulheres, com grau de parentesco próximo (mães, na prática), corroborando que familiares costumam assumir o cuidado ${ }^{(9)}$.

As investigações sobre QV são de grande importância científica e social para subsidiar ações e fomentar políticas sociais, visando a promoção da dignidade e o respeito com os cuidadores e demais segmentos da população $0^{(10)}$.

Com este estudo foi possível mensurar o efeito que o papel de cuidador exerce sobre a QV do próprio cuidador que, comparada com a da população em geral, se mostra diminuída. Os domínios mais afetados nessa relação são os de Dor, Aspectos Físicos, Aspectos Sociais e Aspectos Emocionais, caracteri-

zando a maior demanda física e emocional presente na população de cuidadores de sujeitos com sequelas neurológicas.

Descrever a relação entre a dependência funcional dos sujeitos e a QV de seus cuidadores mostra-se importante, pois contribui para a visualização das reais necessidades dessa população, particularmente quando se planeja ações em saúde. Desta forma, o processo de intervenção dos profissionais de saúde deve também contemplar aquelas ações voltadas à realidade apresentada no presente estudo: a atenção aos cuidadores, para que sejam minimizados os problemas que poderão advir da condição de QV diminuída.

O cuidador familiar desconhece como proceder com quem recebe os cuidados, pois este envolve uma ampla reformulação cotidiana, financeira, de afeto, de organização do espaço e das atribuições dos membros da família ${ }^{(5)}$. Estes fatores se diferenciam conforme 0 nível de dependência do sujeito que será cuidado. Os dados mostram a necessidade de uma abordagem específica sobre a QV de cuidadores de sujeitos com completa dependência, pois esses cuidadores apresentaram a QV piorada em relação aos cuidadores de sujeitos nos demais níveis; ou seja, quanto maior o grau de dependência do sujeito, pior a QV do cuidador.

Propõem-se, assim, a continuidade do estudo 
aprofundando ações direcionadas aos cuidadores de sujeitos com sequelas de doenças neurológicas, com foco principal para os cuidadores de sujeitos que apresentam nível de dependência completa.

\section{REFERÊNCIAS}

1. Mion Júnior D, Gusmão JL, Pierin AMG. Avaliação da qualidade de vida do paciente hipertenso: proposta de um instrumento. Cartilha do hipertenso, v1, n1, 2005.

2. Silva MLP, Henriques MERM. Qualidade de vida de idosos acamados em um município paraibano. Cogitare Enferm. 2008;13(1):140-1.

3. Riberto M, Miyazaki MH, Jorge Filho D, Sakamoto H, Battistella LR. Reprodutibilidade da versão brasileira da medida de independência funcional. Acta Fisiátrica. 2001;8(1):45-52.

4. Castro ED, Lima EMFA, Brunello MIB. Atividades humanas e terapia ocupacional. In: De Carlo MMRP, Bartalotti CC, organizadores. Terapia ocupacional no Brasil: fundamentos e perspectivas. São Paulo:Plexus;2001. p.41-59.

5. Bicalho CS, Lacerda MR, Catafesta F. Refletindo sobre quem é o cuidador familiar. Cogitare Enferm. 2008;13(1):118-23.

6. Machado ALG. A vivência do cuidador familiar de pessoa incapacitada por acidente vascular cerebral: uma abordagem interacionista [dissertação]. Fortaleza (CE):Universidade Estadual do Ceará; 2008.

7. Ciconelli RM, Ferraz MB, Santos W, Meinão L, Quaresma MR. Tradução para a língua portuguesa e validação do questionário genérico de avaliação de qualidade de vida SF-36 (Brasil SF-36). Rev Bras Reumatol. 1999;39(3):143-50.

8. Morales RR, Morales NMO, Rocha FCG, Fenelon SB, Pinto RMC, Silva CHM. Qualidade de vida em portadores de esclerose múltipla. Arq Neuro-Psiquiatr. 2007; 65(2-B):454-60.

9. Lacerda MR, Przenyczka KA. Exercício (I)legal da enfermagem: a realidade do cuidador informal. Cogitare Enferm. 2008;13(3):343-51.

10. Pinotti S, Mantovani MF, Giacomozzi LM. Percepção sobre a hipertensão arterial e qualidade de vida: contribuição para o cuidado de enfermagem. Cogitare Enferm. 2008;13(4):526-34. 